\title{
Tétanos du post-partum sur déchirure vaginale à propos d'un cas à l'Hôpital National de Zinder, Niger
}

\section{Mahamadou Doutchi}

Service de Maladies infectieuses et parasitaires, Hôpital National de Zinder,

Faculté des Sciences de la Santé, Université de Zinder, Niger.

BP : 155 ou 656

\section{Harissou Adamou}

Service de chirurgie générale B, Hôpital National de Zinder, Faculté des

Sciences de la Santé, Université de Zinder, Niger

Ibrahim Amadou Magagi

Service de chirurgie générale B, Hôpital National de Zinder-Niger

Amadou Magagi

Service d'Anesthésie-Réanimation, Hôpital National de Zinder-Niger

\section{Souleymane Oumarou Garba}

Gynécologue-obstétricien, Centre Régional Mère-enfant de Zinder-Niger

\section{Abdoul Azize Garba}

Service de médecine interne, Hôpital National de Zinder-Niger

doi: 10.19044/esj.2017.v13n12p301 URL:http://dx.doi.org/10.19044/esj.2017.v13n12p301

\begin{abstract}
We report an exceptional case of postpartum tetanus occurring in an unvaccinated primipara having given birth in a health center. Six days after childbirth, she had a trismus associated with generalized muscle contractions suggestive of tetanus. Obstructed labor was complicated by a vulvar tear repaired with wires under septic conditions. Functional sequelae, such as dysarthria, quadriesteria, and a decrease in visual acuity, marked the evolution. The newborn was free from neonatal tetanus. Our patient poses the problem of access to preventive care and the quality of care in health facilities in developing countries.
\end{abstract}

Keywords: Tetanus, postpartum, vaginal tear, vaccination, Niger

\section{Résumé}

Nous rapportons un cas exceptionnel de tétanos du post-partum survenant chez une primipare non vaccinée ayant accouché dans un centre de santé. Six jours après l'accouchement, elle avait un trismus associé à des 
contractures musculaires généralisées évoquant le diagnostic de tétanos. L'accouchement dystocique était compliqué d'une déchirure vulvaire réparée avec des fils dans des conditions septiques. Des séquelles fonctionnelles à type de dysarthrie, de quadriparésie et d'une baisse de l'acuité visuelle émaillaient l'évolution. Le nouveau-né était indemne de tétanos néonatal. Notre malade pose le problème d'accès aux soins préventifs et de la qualité de la prise en charge dans les formations sanitaires des pays en développement.

Mots-clés : Tétanos, postpartum, déchirure vaginale, Vaccination, Niger

\section{Introduction}

Le tétanos est une toxiinfection, non contagieuse et non immunisante, commune à l'homme et aux animaux, due à un agent microbien anaérobie strict: le Clostridium tétani ou bacille de Nicolaier, commensal du tube digestif de plusieurs espèces animales (Thwaites et al. 2015). Ce germe ubiquitaire pénètre dans l'organisme par effraction, habituellement par une plaie souillée même minime (Ndour et al. 2002, Manga et al. 2009). Il fait partie des 9 maladies cibles du programme élargi de vaccination au Niger. Le tétanos obstétrical est défini comme tous cas de tétanos à porte d'entrée génitale survenu dans les six semaines suivant la fin d'une grossesse sans préjuger des conditions dans lesquelles cette grossesse s'est terminée (Ndour et al., 2002, Manga et al., 2009, Doret et Marcellin., 2015). Il comprend le tétanos post-partum résultant de manœuvres septiques lors de la délivrance et le tétanos post-abortum, que l'avortement soit spontané ou le plus souvent provoqué (Ndour et al. 2002). Le tétanos du post-partum est rare chez les femmes qui accouchent en maternité, mais beaucoup plus fréquent chez celles qui accouchent à domicile (Ndour et al. 2002, Manga et al. 2010). Nous rapportons un cas exceptionnel de tétanos du post-partum généralisé chez une femme non vaccinée ayant accouchée dans une formation sanitaire.

\section{Observation}

Il s'agissait d'une femme de 17 ans, primigeste, primipare, sans antécédent médical particulier, admise au service des maladies infectieuses de l'Hôpital National de Zinder, référée de l'hôpital de district de Mirriah, situé à environ $20 \mathrm{~km}$ de Zinder, pour trismus et contracture généralisée au sixième jour du post partum. La patiente n'avait pas effectué de consultation prénatale et n'était pas vaccinée. Cette patiente avait accouché dans un centre de santé intégré situé à $50 \mathrm{~km}$ de Zinder, par voie basse, à terme, d'un enfant né vigoureux à la naissance, assistée par une infirmière. L'accouchement dystocique, se serait déroulé sans la réalisation d'une épisiotomie. Une déchirure périnéale postérieure intéressant la peau ano- 
vulvaire, les muscles superficiels du périnée et la muqueuse du vagin (Figure 1).

Six jours après l'accouchement survenaient des crises convulsives tonicocloniques généralisées, associée à une fièvre à $38^{\circ} 5 \mathrm{C}$ motivant son hospitalisation. La tension artérielle était normale $(12 / 7 \mathrm{cmHg})$ et la pulsation à 104/min. L'examen clinique retrouvait une déchirure vulvaire surinfectée, l'utérus était tonique et les lochies normales. La patiente présentait un trismus et des contractures musculaires généralisée avec des renforcements paroxystiques du tonus survenant spontanément ou à l'occasion de stimuli souvent minimes (lumière, bruit, soins médicaux).

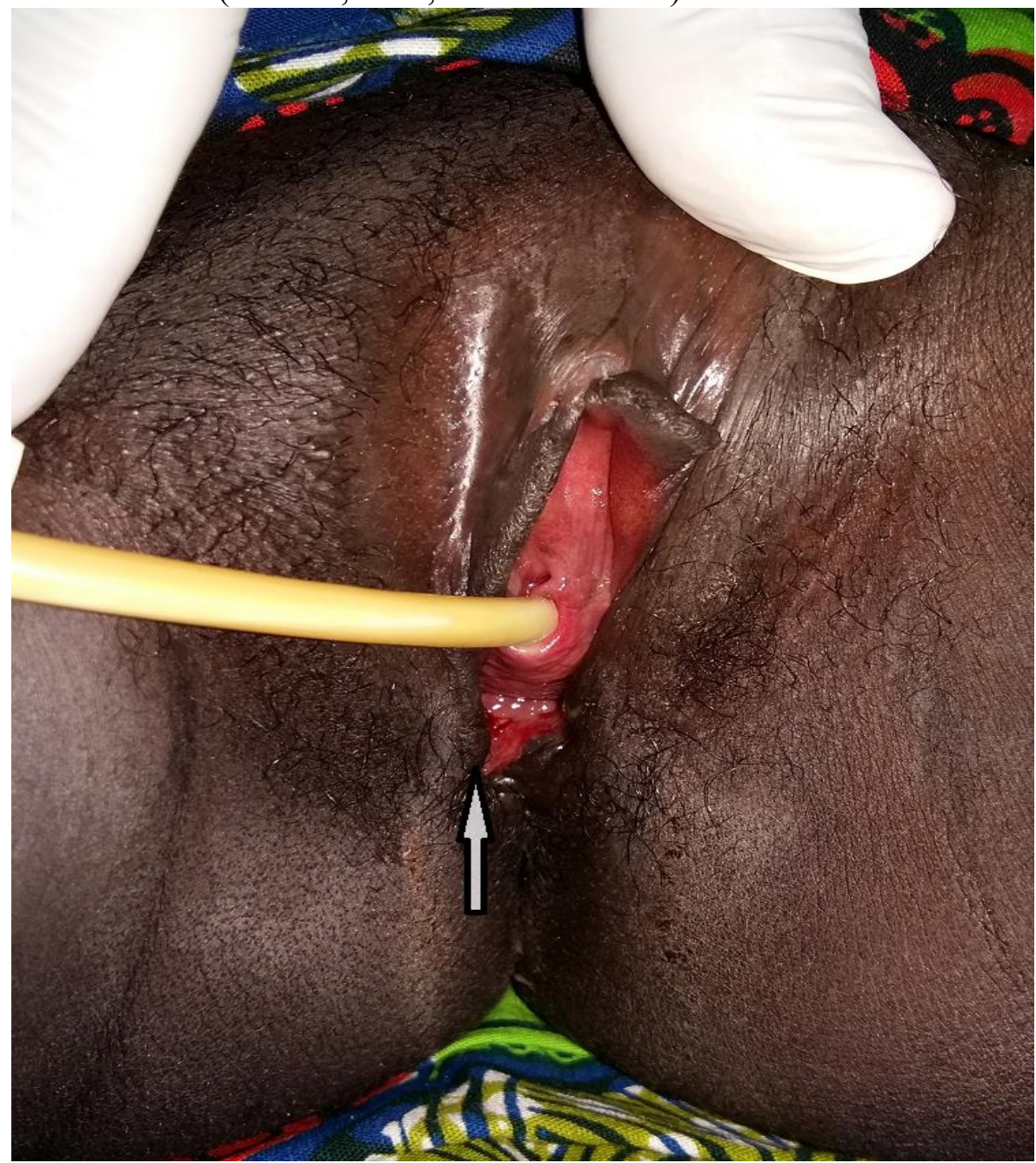

Figure 1 : Aspect de la déchirure vaginale après le parage chirurgical six jours après l'accouchement (flèche).

Elle était raide avec une attitude en opisthotonos : ventre de bois, membres supérieurs fixés en flexion, les membres inférieurs en extension (Figure 2). Les réflexes ostéo-tendineux etaient vifs sans signe de Babinski. La patiente 
présentait des poussées tensionnelles, des accès de tachycardie, de sueurs profuses et de la fièvre.

Le bilan étiologique initial montrait un syndrome inflammatoire biologique (hyperleucocytose à 22000, CRP à 92) et une anémie inflammatoire à 9g/dl. Les hémocultures étaient négatives et la culture sur écouvillon était polymicrobienne : Pseudomonas aeruginosa, candida albicans, Xanthomonas maltophilia, Staphylocoque aureus. La goutte épaisse était positive.

La prise en charge chez notre patiente avait consisté en un traitement étiologique comprenant une biantibiothérapie (métronidazole et ciprofloxacine : $30 \mathrm{mg} / \mathrm{kg} / \mathrm{jour}$ ), une sérothérapie antitétanique (sérum hétérologue d'origine équine ou SAT, 1500 UI en injection intramusculaire), une vaccinothérapie antitétanique ou VAT ( 1 dose en sous-cutané à répéter ultérieurement pour compléter l'état vaccinal). Un traitement antipaludique (quinine $25 \mathrm{mg} / \mathrm{kg} / \mathrm{jour}$ ) était administré. Une prise en charge de la porte d'entrée par un parage chirurgical, ablation des fils de sutures, des corps étrangers et des soins locaux quotidiens suivi de toilette périnéale biquotidienne avec une solution d'antiseptique (éosine aqueuse $2 \%$ ) étaient réalisés. Le traitement symptomatique comportait un isolement sensoriel, une administration de sédatifs et de myorelaxants (diazépam : $10 \mathrm{mg} / \mathrm{kg} / \mathrm{jour}$ en perfusion dans du sérum glucosé, phénobarbital : 1 à $2 \mathrm{cg} / \mathrm{kg} /$ jour en 1 injection IM et le thiocolchicoside: $8 \mathrm{mg} / \mathrm{j}$ en IM). Par ailleurs, nous avons utilisé du Lovenox 6000 UI en injection sous-cutanée pour la prévention de la maladie thromboembolique ainsi que des mesures hygiéno-diététiques (hydratation suffisante, alimentation suffisante et équilibrée, nursing, soins de propreté, lutte contre les escarres).

L'apyrexie n'était obtenue qu'à J15. Le nouveau-né était indemne de tétanos néonatal. Au bout de 47 jours d'hospitalisation, la patiente était sortie de l'hôpital, déclarée guérie mais avec des séquelles fonctionnelles représentées par une dysarthrie, une quadriparésie, une baisse de l'acuité visuelle et une amyotrophie avec amaigrissement de $14 \mathrm{~kg}$ par rapport à son poids d'admission. Une rééducation fonctionnelle était prescrite après la sortie de l'hôpital. 


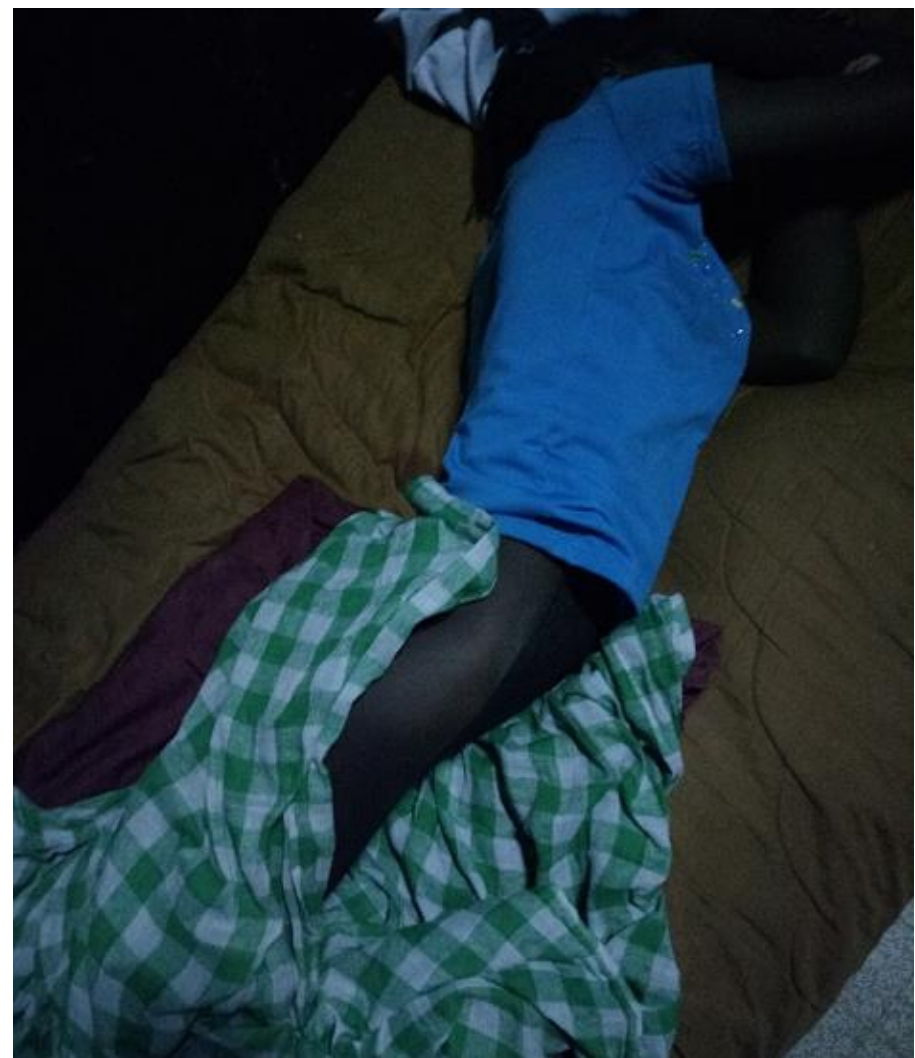

Figure 2 : Attitude en opisthotonos de la patiente.

\section{Discussion}

Le tétanos demeure un problème de santé publique dans les pays en développement malgré l'existence du programme mondiale d'immunisation qui a prévu son élimination pour 2005 (Thwaites et al. 2015, Wateba et al.2008). Dans les pays développés, le tétanos est devenu une maladie rare, survenant essentiellement chez les sujets âgés qui ont échappé à la vaccination, ou qui sont insuffisamment immunisés (Thwaites et al. 2015, Khan et al. 2015). La grande majorité des pays n'ayant pas encore éliminé le tétanos maternel et néonatal est constitué par les 50 pays les plus pauvres du monde. Le nombre de cas de tétanos maternel est estimé entre 15000 et 30000 par an dans le monde (Manga et al. 2009).

$\mathrm{Au}$ Niger, la vaccination des femmes enceintes fait partie des prestations offertes au cours des consultations prénatales dans les centres de santé publics. La couverture vaccinale des femmes enceintes en 2015 était de $61 \%$ (SNIS-Niger, 2015). Le tétanos n'est donc pris en compte que dans le cadre de l'immunisation des femmes enceintes et des enfants de 0 à 6 mois. La couverture vaccinale des femmes enceintes en 2015 était de 61\% (SNISNiger, 2015). Cette mesure restrictive semble insuffisante pour lutter 
efficacement contre cette affection dont seule la vaccination systématique et généralisée permettrait d'envisager l'éradication. Aussi, après un accouchement assisté, une réparation chirurgicale dans les conditions aseptiques d'une déchirure vaginale, une sérothérapie antitétanique aurait minimisée le risque de développer un tétanos obstétrical.

Clostridium tétani peut être isolé à partir du site d'entrée (Thwaites et al. 2015). Lorsque le taux d'IgG antitétanique plasmatique est supérieure à 0,1 $\mathrm{UI} / \mathrm{mL}$ (prélevée avant l'administration d'antitoxine), le diagnostic de tétanos est moins probable car constituant une protection suffisante contre l'infection (Thwaites et al. 2015, Wateba et al.2008, Khan et al. 2015). La détection par PCR de la toxine tétanique dans les exsudats plasma peut être aussi utilisée dans le diagnostic (Thwaites et al. 2015).

Chez notre patiente, le diagnostic de tétanos était posé sur la base d'un ensemble d'arguments anamnestiques et cliniques tels que : l'absence de vaccination antitétanique à jour, la mise en évidence d'une porte d'entrée (déchirure vulvaire suturée et surinfectée), la présence d'un trismus avec signe de l'abaisse-langue captif d'Armengaud, d'une contracture généralisée avec attitude en opisthotonos et des paroxysmes spontanés. Aucun argument biologique n'était nécessaire pour le diagnostic. Tout trismus doit être considéré comme étant un tétanos jusqu'à preuve du contraire (Thwaites et al. 2015, Ndour et al. 2002, Manga et al. 2009). Dans la plupart des cas, le tétanos maternel se présente sous forme généralisée. Les périodes d'incubation chez les enfants plus âgés et les adultes sont habituellement plus longues que chez les nouveau-nés, avec des moyennes de 7 à 10 jours (intervalle 2-38 jours). Plus celle-ci est courte, plus la maladie est sévère (Thwaites et al. 2015). Le temps d'incubation de 6 jours associé à une porte d'entrée gynécologique et la présence des paroxysmes classe notre patiente dans les formes graves de tétanos selon le score pronostique de Dakar (Ndour et al. 2002, Manga et al. 2009). Dans une étude sur une série de 21 cas de tétanos obstétrical, Ndour et al. 2002, retrouvaient 85,71\% de formes de moyenne gravité. Dans la même série, ces auteurs rapportaient un délai d'admission de 2,4 jours. Chez notre patiente le délai d'admission était de 24 heures. La présence de la fièvre chez notre patiente peut être un signe de gravité de son tétanos ou lié à un accès palustre. En effet, la présence de la fièvre est un signe de gravité du tétanos et doit faire suspecter une complication (Pilaca et al. 2012).

Le traitement du tétanos consiste habituellement, en une association de sérothérapie et de vaccination antitétanique, d'antibiothérapie, de sédatifs, de myorelaxants, de soins de la porte d'entrée, d'hydratation et d'alimentation par sonde nasogastrique (Thwaites et al. 2015, Ndour et al. 2002, Manga et al. 2009, Doret et al. 2015, Khan eta al. 2015, Pilaca et al. 2012, Abbate et al. 2008). 
La pénicilline ou le métronidazole par voie intraveineuse sont des traitements de première intention du tétanos maternel (Thwaites et al. 2015, Wateba et al.2008). L'injection par voie parentérale de l'antitoxine antitétanique réduit la mortalité. L'administration d'antitoxine via la voie intrathécale est utilisée depuis plusieurs années (Thwaites et al. 2015, Khan et al. 2015). Elle constitue un moyen pour neutraliser la toxine dans le système nerveux et augmente la concentration intrathécale de l'antitoxine par rapport aux concentrations obtenues après administration intramusculaire (Khan et a al. 2015, Wateba et al.2008). Elle donne un meilleur résultat en termes de réduction de la durée d'hospitalisation et de sévérité des symptômes (Thwaites et al. 2015, Wateba et al.2008, Khan et al. 2015). Dans de nombreux pays en développement, la chlorpromazine et le phénobarbital sont les piliers du traitement symptomatique du tétanos, car très abordables (Thwaites et al. 2015).

Notre patiente est sortie guérie, mais avec d'importantes séquelles neurologiques et ostéoarticulaires à type de dysarthrie, de tétraparésie et de douleurs ostéo articulaires. Ces séquelles ont été décrites dans les formes graves de tétanos (Thwaites et al. 2015, Manga et al. 2010, Khan et al. 2015, Abbate et al 2010). Le tétanos maternel issu de sites d'entrée internes (postpartum, postabortum) est associé à une mortalité élevée. Des taux de mortalité allant jusqu'à $52 \%$ ont été rapportés en Asie et en Afrique (Thwaites et al. 2015).

Au Niger, L'efficacité du programme élargi de vaccination (PEV) en termes d'accessibilité et la limitation de la prévention du tétanos aux femmes enceintes et aux enfants de moins d'un an est un problème majeur. En effet une extension de la cible aux femmes en âge de procréer aurait permis d'atteindre une population plus importante, souvent ratée lors des grossesses.

La prévention et l'élimination du tétanos maternel et néonatal ne peut être obtenues qu'en combinant la vaccination et l'amélioration de la qualité des soins obstétricaux et périnataux (Thwaites et al. 2015).

\section{Conclusion}

Le tétanos du post partum est une pathologie grave, potentiellement mortelle. Il survient habituellement suite à un accouchement septique à domicile ou dans des structures sanitaires à hygiène précaire. La campagne d'immunisation antitétanique au Niger exclut une frange importante des femmes en âge de procréer. Une révision du PEV et une amélioration de la qualité des soins dans les formations sanitaires du pays est indispensable pour éradiquer le tétanos obstétrical.

Conflit d'intérêts : aucun 


\section{References:}

1. Thwaites CL, Beeching NJ, Newton CR (2015). Maternal and neonatal tetanus. The Lancet.385 (9965):362-70.

2. Ndour CT, Soumaré M, Diop BM, Touré AK, Badiane S (2002). Le tétanos à porte d'entrée obstétricale à Dakar: aspects épidémiologiques et pronostiques à propos de 21 cas observés à la clinique des maladies infectieuses du CHU de Dakar. Médecine Mal Infect.32(8):399-404.

3. Manga NM, Ndour CT, Fortes L, Diop SA, Dia NM, Mbaye M, et al (2009). Le tétanos de la femme en âge de procréer à la clinique des maladies infectieuses de Dakar. Bull Soc Pathol Exot.;102(4):221-225.

4. Doret M, Marcellin L (2015.). Les vaccinations dans le postpartum immédiat: recommandations. J Gynécologie Obstétrique Biol Reprod; 44(10):1135-1140.

5. Manga NM, Dia NM, Ndour CT, Diop SA, Fortes L, Faye A, et al (2010). Maternal tetanus in Dakar from 2000 to 2007. Med Trop Rev Corps Sante Colon; 70 (1):97-8.

6. Wateba M, Diop S, Nichols S, Patassi A, Adjo S, Gbadamassi G, et al (2008). Intérêt de la thérapie intrathécale à 1500 UI de sérum antitétanique combinée à 1,5 gramme de métronidazole en intraveineux sur le pronostic du tétanos en milieu hospitalier togolais. Cahiers Santé; 18(3):125-9.

7. Khan R, Vandelaer J, Yakubu A, Raza AA, Zulu F (2015). Maternal and neonatal tetanus elimination: from protecting women and newborns to protecting all. 2015. Int J Womens Health; 3; 7:171-80. doi: 10.2147/IJWH.S50539.

8. SNIS-Ministère de la Sante Publique. Republique du Niger. Annuaire des Statistiques Sanitaires du Niger. (2015). http://www.stat-niger.org/statistique/index.php

9. Pilaca AS, Beqiri AI, Ndreau A, Puca ES, Pepa AK, Elezi FM (2012). Factors affecting the prognosis of Albanian adult patients with generalized tetanus.. Il G Chir; 33(4):105-9.

10. Abbate R, Di Giuseppe G, Marinelli P, Angelillo IF, Group CW. (2008). Appropriate tetanus prophylaxis practices in patients attending Emergency Departments in Italy. Vaccine; 26(29):36343639. 